\title{
Hernioplastia inguinal de Lichtenstein, experiencia en el Hospital Básico \\ IESS Chone.
}

\author{
Lichtenstein inguinal hernioplasty, experience at IESS Chone Basic \\ Hospital.
}

\section{Hernioplastia inguinal de Lichtenstein}

\author{
Orelvis Rodríguez Palmero ${ }^{(1)}$ \\ Liseidy Ordaz Marín ${ }^{(2)}$ \\ Agustín Marcos García Andrade ${ }^{(3)}$ \\ María Del Rosario Herrera Velázquez ${ }^{(4)}$
}

(1) Hospital Básico IESS Chone, docente del ITSUP; orelvis70@gmail.com

(2) Hospital Básico MSP Jipijapa; liseidyordaz@gmail.com

(3) Hospital Básico IESS Chone, director médico; agustinmarcosg@yahoo.com

(4) Carrera de enfermería de la Universidad Estatal del Sur de Manabí. UNESUM; vehemari@ gmail.com

Contacto: orelvis70@gmail.com

Recibido: 18-02-2020 Aprobado:28-02-2020

\section{Resumen}

La hernia inguinal continúa siendo un desafío para los cirujanos a pesar de la aplicación de técnicas libres de tensión con el uso de mallas, las tasas de recidivas siguen siendo altas. Objetivo: Evaluar los resultados de la aplicación de la técnica de reparación libre de tensión de Lichtenstein. Métodos: Se realizó un estudio descriptivo, prospectivo y transversal en 215 pacientes con diagnóstico de hernia inguinal, desde agosto 2017 a agosto 2019, en el Hospital Básico del Instituto Ecuatoriano de Seguridad Social de Chone, Manabí, Ecuador. Resultados: Fueron operados 215 pacientes, el rango de edad con mayor número de pacientes fue el de 50 a 59 con 71 casos, el sexo masculino reporta el $95 \%$ de los casos, para la clasificación de las hernias se utilizó la de Nyhus, las de tipo IIIb de esta clasificación fue la de mayor predominio con el $54.3 \%$, en cuanto a localización de la hernia y su forma clínica de presentación, el lado derecho fue el más afectado con 127 casos, y la forma clínica la hernia primaria con $96.2 \%$, el seroma de la herida se presentó como complicación en 11 pacientes.

Palabras clave: Hernia inguinal; Hernioplastia; malla; técnica de Lichtenstein.

Abstract
Inguinal hernia continues to be a challenge for surgeons despite the application of stress-free techniques with the use of meshes, relapse rates remain high. Objective: To evaluate the results of the application of Lichtenstein's stress-free repair technique. Methods: A descriptive, prospective and cross-sectional study was conducted in 215 patients diagnosed with inguinal hernia, from August 2017 to August 2019, at the Basic Hospital of the Ecuadorian Social Security Institute of Chone, Manabí, Ecuador. Results: 215 patients were operated, the age range with the highest number of patients was 50 to 59 with 71 cases, the male sex reports $95 \%$ of cases, for the classification of hernias was used that of Nyhus, the type IIIb of this classification was that of the highest prevalence with $54.3 \%$, in terms of location of the hernia and its clinical form of presentation, the right side was the most affected with 127 cases, and the clinical form of primary hernia with $96.2 \%$, was high blood pressure the highest weighting functionality with 46 seroma from the wound was presented as a complication in 11 patients.

Keywords: Inguinal hernia; Hernioplasty; mesh; Lichtenstein's technique.

\section{Introducción}

La hernia inguinal como enfermedad es tan vieja como la humanidad misma (Valeis, 2019). En su formación se invocan varios factores, entre los que se destacan la debilidad de la pared abdominal para 
contener los órganos intrabdominales, factores genéticos y adquiridos. Los estudios de microscopia electrónica han demostrado una disminución de las fibras oxitalánicas de la colágena, con un incremento en la sustancia amorfa de las fibras elásticas, lo que produce una severa alteración de la resistencia de la fascia transversalis (Rodríguez, 1990).

Los siglos XIX y XX vieron florecer infinidad de técnicas ideadas y empleadas con el objetivo de reparar los defectos herniarios de la región inguinocrural, todas basadas en el principio básico del uso de suturas para reparar el defecto mediante la aproximación de los tejidos implicados con mejores, peores y hasta desalentadores resultados. A la luz de los conocimientos actuales la causa principal de fracaso en todas las hernioplastias en que el cierra el orificio miopectíneo por aproximación aponeurótica, es la tensión (Pérez-Suárez, 2015).

Fue Celcius el primero en proponer una técnica para la reparación de las hernias (Montejo, 2011). Eduardo Bassini (1844- 1924) invoca la reparación de la pared posterior del canal inguinal como técnica quirúrgica (Caraballoso, 2016).

La aparición de las técnicas libre de tensión, dentro de las cuales se destaca la de Lichtenstein, han tenido gran aceptación y avance, logando bajar las tasas de recidiva hasta un $2 \%$ en promedio (Cuenca, 2015). La técnica de Lichtenstein consiste en la colocación de una prótesis de malla para cumplir con tres principios básicos en la reparación herniaria: endurecer el peritoneo del saco herniario, efecto de tapón y formar una pared nueva en la cicatrización sobre la malla (Castro, 2018).

En este sentido se han empleado otras vías como la laparoscópica para la reparación de las hernias de la pared abdominal, utilizando diferentes tipos de mallas, con un mínimo de complicaciones y con una baja tasa de recidivas (Herrera, 2017)

La selección de las técnicas quirúrgicas para la reparación de las hernias inguinales depende de las características de la hernia en su clasificación clínica, de la experiencia, habilidad y el desempeño del cirujano, no creemos que una técnica supere a la otra, no existe un procedimiento estándar, la diversidad de técnicas hace referencia por si sola a lo complejo de la reparación de las hernias de la pared abdominal. Si bien todo lo anterior es cierto la técnica de reparación descrita y desarrollada por Irving Lichtenstein, cumple con los requisitos de una técnica sencilla, fácil de aprender y desarrollar, que ofrece una baja tasa de recidivas.

En el Hospital Básico del Instituto Ecuatoriano de Seguridad Social de Chone se ha logrado estandarizar la técnica de Lichtenstein en la reparación de las hernias inguinales, por lo que motivados por el avance en técnicas de reparación y la no existencia de estudios previos en la institución, se decidió realizar este trabajo con el objetivo de evaluar el resultado de esta técnica en el Hospital Básico IESS de Chone.

El presente trabajo tiene por objetivo evaluar los resultados de la aplicación de la técnica de reparación libre de tensión de Lichtenstein, en el Hospital Básico del Instituto Ecuatoriano de Seguridad Social de Chone, Manabí, Ecuador.

\section{Materiales y métodos}

Se realizó un estudio descriptivo, prospectivo y transversal en 215 pacientes con diagnóstico de hernia inguinal, operados por la técnica de Lichtenstein, en el periodo comprendido desde agosto 2017 a agosto 2019, en el Hospital Básico del Instituto Ecuatoriano de Seguridad Social de Chone, Manabí, Ecuador.

En el estudio se incluyeron todos los pacientes desde los 18 años con diagnóstico de hernia inguinal y que fueron operados con la técnica antes descrita, los datos para cada una de las variables fueron obtenidos del sistema informático AS400, se delimitaron como variables la edad, en años cumplidos, sexo, tipo de hernia según la clasificación de Nyhus, localización de la hernia y su forma clínica de presentación, así como las complicaciones, la que incluyen las recidivas herniarias.

Para la reparación herniaria se utilizó una prótesis prediseñada de Fluoruro de polivinilideno (PVDF) DynaMesh®-LICHTENSTEIN de fabricación alemana. Es un filamento con muchas propiedades «naturales» positivas. La segunda garantía la constituyen las estructuras tricotadas de tejido a partir de dicho filamento. Los filamentos de PVDF poseen una excelente biocompatibilidad lo que conlleva a una escasa reacción a cuerpos extraños, evitándose de forma segura la formación de placas cicatriciales, contribuyendo así a un mayor confort para el paciente. Así, el PVDF, muestra una formación de granuloma significativamente menor (tejido cicatricial), en comparación con los polímeros convencionales. DynaMesh®-LICHTENSTEIN posibilita un manejo operatorio seguro y con ahorro de tiempo. La elasticidad obtenida gracias a una técnica especial de entramado del tejido facilita notablemente la colocación de la malla sin pliegues (Gerullis, 2012). 


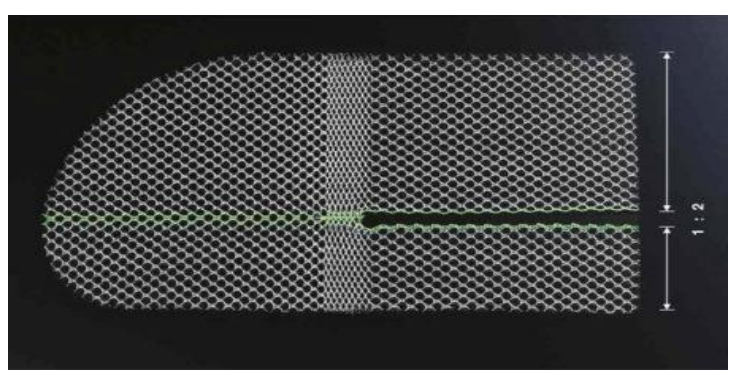

Figura. 1. Malla prediseñada de DynaMesh®LICHTENSTEIN.

Se procedió al diseño de una base de datos para poder realizar el análisis estadístico, se utilizó el programa Microsoft Office Excel. Se estimaron los porcentajes como medida de resumen de la estadística descriptiva y se estimaron las frecuencias relativas.

La investigación se realizó bajo los principios de la ética médica. Se aplicaron las normas de las investigaciones científicas aplicadas a documentos médicos. A todos los pacientes y sus familiares, previo al acto operatorio, en consulta preoperatoria de cirugía se les solicitó la firma del consentimiento informado, cumpliendo con las normas hospitalarias de cirugía segura.

\section{Resultados}

En el período de agosto del 2017 a agosto 2019 se operaron un total de 215 pacientes. La edad y sexo de los pacientes fueron variables fundamentales para poder conocer el comportamiento de la hernia inguinal en el hospital donde se realizó este estudio. En el estudio predominó el sexo masculino con 204 pacientes, para el $(95 \%)$, en cambio el femenino solo se reportó 11 casos para el (5\%). Los pacientes con el rango de edad entre los 50 a 59 años fueron los más afectados con 71 casos, para el (32.9\%); seguido por el rango de 30 a 49 con 64 pacientes para el (30\%). Es preciso señalar que en el estudio se presentaron 9 pacientes mayores de 80 años para el (4.1\%), todos del sexo masculino, (Tabla No 1)

\begin{tabular}{|l|c|c|c|c|}
\hline \multirow{2}{*}{ Edad } & \multicolumn{2}{|c|}{ Masculino } & \multicolumn{2}{c|}{ Femenino } \\
\cline { 2 - 5 } & $\mathbf{N}^{\mathbf{0}}$ & $\mathbf{\%}$ & $\mathbf{N}^{\mathbf{0}}$ & $\mathbf{\%}$ \\
\hline $18-29$ & 22 & 10.2 & 1 & 0.4 \\
\hline $30-49$ & 60 & 28 & 4 & 2 \\
\hline $50-59$ & 70 & 32.5 & 1 & 0.4 \\
\hline $60-69$ & 33 & 15.3 & 3 & 1.3 \\
\hline $70-79$ & 10 & 5 & 2 & 0.9 \\
\hline 80 y mas & 9 & 4.1 & 0 & 0 \\
\hline Total & 204 & 95 & 11 & 5 \\
\hline
\end{tabular}

Tabla 1: Distribución de pacientes según edad y sexo. IESS Chone.

Fuente: Sistema AS400. IESS CHONE.
Para la clasificación de las hernias de la región inguinocrural se utilizó la de Nyhus, las de tipo IIIb predominaron con 117 pacientes $(54.3 \%)$ estas son hernias indirectas que protruyen a través de anillo inguinal profundo, afectando también la pared inguinal posterior, donde el contenido puede ocupar totalmente el canal inguinal y en muchos casos progresar y descender hasta el escroto o convertirse en una verdadera hernia en pantalón, con dos sacos uno indirecto y otro directo a ambos lados de los vasos epigástricos. Las hernias directas tipo IIIa de esta clasificación reportó 73 casos $(34 \%)$ en estas el saco protruye por debilidad directa de la pared posterior. Se reportaron 4 pacientes operados con recurrencias herniaria para el $(1.7 \%)$ (Tabla No 2)

\begin{tabular}{|c|c|c|}
\hline Clasificación de Nyhus & $\mathrm{N}^{\circ}$ & $\%$ \\
\hline Nyhus II & 21 & 10 \\
\hline Nyhus IIIa & 73 & 34 \\
\hline Nyhus IIIb & 117 & 54.3 \\
\hline Nyhus IVa & 3 & 1.3 \\
\hline Nyhus IVb & 1 & 0.4 \\
\hline Total & 215 & 100 \\
\hline
\end{tabular}

Tabla 2: Tipo de hernia según la clasificación de Nyhus.

Fuente: Sistema AS400. IESS CHONE.

El lado más afectado por hernia en nuestra serie fue el derecho con 127 casos (59\%), el izquierdo con 75 (35\%) y solo se presentaron 13 pacientes con hernias bilaterales (6\%). De las formas clínicas de presentación la primohernia o hernia primaria apareció en el (96.2\%) de los casos, la encarcelada o atascada es aquella en la cual el contenido del saco crea una obstrucción intestinal mecánica sin compromiso de la vascularización del órgano atrapado en el saco, esta apareció en 2 casos (0.9\%) y solo un caso con hernia estrangulada para el $(0.4 \%)$ (Tabla No 3). 


\begin{tabular}{|l|l|l|l|l|l|l|l|l|l|l|l|l|}
\hline Localización & \multicolumn{2}{|c|}{ Primohernia } & \multicolumn{2}{|c|}{ Atascada } & \multicolumn{2}{c|}{ Reproducida } & \multicolumn{2}{|c|}{ Iterada } & \multicolumn{2}{|c|}{ Estrangulada } & \multicolumn{3}{|c|}{ Total } \\
\hline & \multicolumn{1}{|c|}{$\mathbf{N}^{\mathbf{0}}$} & $\mathbf{\%}$ & $\mathbf{N}^{\mathbf{0}}$ & $\mathbf{\%}$ & $\mathbf{N}^{\mathbf{0}}$ & $\mathbf{\%}$ & $\mathbf{N}^{\mathbf{0}}$ & $\mathbf{\%}$ & $\mathbf{\%}$ & $\mathbf{N}^{\mathbf{0}}$ & $\mathbf{\%}$ & $\mathbf{N}^{\mathbf{0}}$ \\
\hline Derecha & 121 & 56.2 & 2 & 0.9 & 3 & 1.3 & 0 & 0 & 1 & 0.4 & 127 & 59 \\
\hline Izquierda & 73 & 33.9 & 0 & 0 & 1 & 0.4 & 1 & 0.4 & 0 & 0 & 75 & 35 \\
\hline Bilateral & 13 & 6 & 0 & 0 & 0 & 0 & 0 & 0 & 0 & 0 & 13 & 6 \\
\hline Total & 207 & 96.2 & 2 & 0.9 & 4 & 1.8 & 1 & 0.4 & 1 & 0.4 & 215 & 100 \\
\hline
\end{tabular}

Tabla 3: Localización de la hernia y forma clínica de presentación.

Fuente: Sistema AS400. IESS CHONE.

En 26 pacientes aparecieron complicaciones para el (12\%), el seroma de la herida se diagnosticó en 11 casos $(5.1 \%)$, el hematoma en 7 pacientes $(3.2 \%)$, la orquitis 5 casos $(2.3 \%)$, el dolor inguinal crónico 2 $(0.9 \%)$, la recidiva herniaria $1(0.4 \%)$. (Tabla No 4$)$

\begin{tabular}{|l|c|c|}
\hline Complicaciones & $\mathrm{N}^{\mathrm{o}}$ & $\%$ \\
\hline Seroma de la herida & 11 & 5.1 \\
\hline Hematoma de la herida & 7 & 3.2 \\
\hline Orquitis & 5 & 2.3 \\
\hline Inguinodinea & 2 & 0.9 \\
\hline Recidiva herniaria & 1 & 0.4 \\
\hline Total & 26 & 12 \\
\hline
\end{tabular}

Tabla 4: Complicaciones.

Fuente: Sistema AS400. IESS CHONE.

\section{Discusión}

El uso de biomateriales en cirugía de reparación de las hernia de la pared abdominal fue en el pasado casi de manera selectiva solo en grandes defectos herniario o en recidivas a partir de técnicas convencionales sin mallas, el avance de las tecnologías en la fabricación de biomateriales ligado a la experiencia en su aplicación han cambiado en los últimos 30 años la manera en que reparamos los defectos herniarios de la pared abdominal, tal es así que hay técnicas que casi se han convertido por si solas en una espacie de gold standard de las reparaciones, como es el caso de la técnica de Linchtestein, descrita por este hace casi 49 años (Lozano, 2012).

La hernia inguinal es una enfermedad que afecta cualquier grupo de edad y ambos sexos, conocer su prevalencia se ha convertido en un gran desafío en algunos estudios se hace referencia a que se desconoce, en otros no es totalmente exacta y varía de región en región, hay estudios que indican 110 a 138 por cada 100000 habitantes (Acevedo, 2006).

La hernia inguinal puede afectar ambos sexos, pero es mucho más frecuente en hombres que en mujeres, el riesgo de desarrollar una hernia inguinal en hombres es de un $26 \%$ y en las mujeres de un $3 \%$, otros trabajos muestran similares resultados, las causas están relacionadas con un mayor grosor en el hombre del anillo inguinal profundo al paso del condón espermático y la menor oblicuidad del canal inguinal (Perero, 2018).

Existen innumerables clasificaciones para las hernias del orificio miopectíneo, están determinadas por varios aspectos de la anatomía de dicha región, entre los cuales se encuentran: tipo de contenido y la posibilidad de reducción por taxia del mismo, grado de progresión del saco, diámetro del anillo inguinal profundo, grado de desplazamiento de los vasos epigástricos, integridad de la pared inguinal posterior, entre otros. La clasificación de Nyhus es una de las más utilizadas, por su sencillez y de fácil guía para selecciona la técnica quirúrgica (Valeis, 2019).

En el estudio predominaron las hernias tipo IIIb de la clasificación de Nyhus, lo que coincide con otros autores (Valeis, 2019) y (Saliou, 2019), sin embargo en la literatura aparecen autores como Pérez Suárez (Pérez, 2015) en el cual las tipo IIIa fueron la de mayor frecuencia.

En cuanto a la forma clínica de presentación, la primohernia permanece como la más frecuente de todas las formas clínicas desde un 80 a un $90 \%$ en las series revisadas (Amid, 2015) y (Shamji, 2010), nuestra serie coincide con el de los autores antes mencionados. El lado derecho se ve más afectado que lado izquierdo con el 60 y el $25 \%$ respectivamente (Torres, 2019).

Las complicaciones que aparecen con la aplicación de la técnica de Lichtenstein no difieren de manera alguna con las reportadas en series donde se aplican otras técnicas protésicas. Las más reportadas son los seromas y los hematomas de la herida quirúrgica (Moreno, 2017), la formación de granulomas por rechazo al material de sutura y las neuralgias. Al ser una técnica de fácil ejecución, que no requiere de gran disección del canal inguinal hace que aparezcan pocas complicaciones que en otras técnicas que si 
requieren de mucha movilización de tejido como el PHS (Prolene hernial system) o el mesh plug.

\section{Bibliografía}

Acevedo AF., Reyes, E., Lombardi, J., Robles, I. (2006) Prevalencia de la cirugía de las hernias inguinales Rev. Chilena de Cirugía. 58 (2), 133-137.

Amid, PK., Shulman, AG., Lichtenstein, IL. (Marzo 2015) Local anesthesia for inguinal hernia repair step-by-step procedure. Ann Surg, 220:735-7. Recuperado de http://www.ncbi.nlm.nih.gov/pmc/articles/P $\underline{\mathrm{MC} 1234473 /}$

Castro García, FJ., Salinas Muñoz, MK. (2018). Frecuencia de recidivas en pacientes sometidos a hernioplastía usando la técnica Lichtenstein en el Hospital Teodoro Maldonado Carbo en el periodo 2006 2016.Universidad Católica de Santiago de Guayaquil. Recuperado de http://repositorio.ucsg.edu.ec/handle/3317/11 $\underline{436}$

Caraballoso García, VJ., Casanova Pérez, PA., Galloso Cueto, GL., González-Chávez, AS., Orea Cordero, I., Caraballoso García, L. (Julio 2016) Resultados del tratamiento quirúrgico de los pacientes operados de hernia inguinocrural Rev. Med. Electrón. 38 (4). Matanzas jul.-ago. 2016

Cuenca Torres, O., Ferreira Acosta R., Martínez, N. (Junio 2015) técnica de separación de componentes con refuerzo de malla de polipropileno para el tratamiento de afecciones parietales complejas. Nuestra experiencia. Rev. cir. parag. 39 (1), asunción june 2015.

Gerullis, H., Georgas, E., Eimer, C., Goretzki, PE., Lammers, BJ., Klosterhalfen, B., Borós, M., Wishahi, M., Heusch, G., Otto, T. (2012) Evaluation of Biocompatibility of Alloplastic Materials: Development of a Tissue Culture in Vitro Test System. Surgical Technology International XXI; CUniversal Medical Press, Inc. USA (2012)

Herrera, HR., Sanabria, R., Llano, E., Pedrozo, A. (2017) Hernia de spiegel no complicada, abordaje laparoscopico. Recuperado de http://revista.medicinauni.edu.py/index.php/F M-uni/issue/view/3

Lozano Corona, R., Beltrán-Estrada, A., Gómez Ríos, N., Rodríguez Ortega, F. (2012). Irving
Lichtenstein, maestro de la cirugía inguinal: semblanza de su vida y obra. Cir. gen 34 (1).

Montejo Saínz, JE., Pisonero Socías, JJ., Delgado Alonso' AI., (2011) Uso de malla de polipropileno en la hernioplastia por técnica de Lichtenstein. Rev. Cubana Cirv.50 (2). Ciudad de la Habanaabr.jun. 2011

Moreno Egea, A. (2013) ¿Es segura la hernioplastia sin sutura como opción para tratar las hernias de pared abdominal? Estudio prospectivo con un adhesivo tisular sintético (n-hexil $\alpha$ cianoacrilato). Rev. Cir. Esp. 91(4): e19-e24.

Pérez Suárez, MJ., Ricardo Martínez, D., Pérez Suárez, CM., Pujol Legrá, PM., Vargas Oliva, JM. (2015) La hernioplastia de Lichtenstein en el tratamiento de la hernia inguinal. Nuestra experiencia. Multimed. Revista Médica. Granma, 19(2) Abril-Junio

Perero Rodríguez, AL., Hurtado Cherrez, TC. (2018) Hernias inguinales: prevalencia $\mathrm{y}$ complicaciones posquirúrgicas en pacientes de 12 a 18 años. Recuperado de http://repositorio.ug.edu.ec/handle/redug/313 $\underline{91}$

Rey -Valeis, Y., Vallés Gamboa, M., Fonseca Sosa, F., Quesada Martínez, E., Núñez Sire, R. (2019) Utilidad de la técnica de Lichtenstein y RutkowRobbins en el tratamiento de la hernia inguinal. Multimed. Revista Médica. Granma, 23(1).

Rodríguez Júnior, AJ., De Tolosa, EM., De Carvalho, CA. (1990). Electron microscopic study on the elastic and elastic related fibres in the human fascia transversalis at different ages. Recuperado de https://www.ncbi.nlm.nih.gov/pubmed/20992 99\#

Saliou Diallo, M., Rodríguez Fernández, Z., Joubert Álvarez, G., Gavilán Yodú, RL., Casamayor Callejas, E. (2019) Factores asociados a las complicaciones de la cirugía electiva de las hernias inguinales. Revista cubana de medicina military, 48(4):707-722.

Shamji, M. F. (2010) What do we gain and lose from database studies? Arch Surg. 145(3):253-254.

Torres Jaramillo, ZV., Paredes Bucheli, DA., Rueda Ruiz, AS., Mena Alencastro, PF., Santos Rivas, LB., Sosa Jurado, JB. (Abril 2019) Tratamiento quirúrgico en pacientes pediátricos con problemas de hernia inguinal. 
Revista Científica Mundo de la Investigación y el Conocimiento, 3 (2)2, ISSN: 2588-073X, 2019, pp. 1248-1265. 